Meta

Journal des traducteurs

Translators' Journal

\title{
Essai de redéfinition du concept de traduction
}

\section{Jacky Martin}

Volume 27, numéro 4, décembre 1982

URI : https://id.erudit.org/iderudit/003588ar

DOI : https://doi.org/10.7202/003588ar

Aller au sommaire du numéro

Éditeur(s)

Les Presses de l'Université de Montréal

ISSN

0026-0452 (imprimé)

1492-1421 (numérique)

Découvrir la revue

Citer cet article

Martin, J. (1982). Essai de redéfinition du concept de traduction. Meta, 27(4), 357-374. https://doi.org/10.7202/003588ar d'utilisation que vous pouvez consulter en ligne.

https://apropos.erudit.org/fr/usagers/politique-dutilisation/ 


\section{ESSAI DE REDÉFINITION DU CONCEPT DE TRADUCTION}

JACKY MARTIN

Avant d'aborder les problèmes théoriques et pratiques de la traduction, il convient d'explorer la littérature particulièrement abondante en ce domaine. Il ne s'agit pas d'établir une bibliographie exhaustive mais de dégager les dominantes du discours contemporain sur la traduction. Récemment, E. Cary déclarait publiquement: "Nous vivons 'l'âge de la traduction' " ${ }^{1}$, mais on pourrait ajouter que si, effectivement, la traduction s'est généralisée comme pratique et même comme concept (en biologie génétique, notamment), la réflexion qui lui est consacrée est encore fort éparpillée, fragmentaire dans ses conclusions, éclectique dans ses principes méthodologiques.

On distingue clairement deux types de recherches : des études empiriques (de loin les plus nombreuses) et des études systématiques. On s'étonnera, sans doute, que nous placions parmi les premières des 'théoriciens' comme G. Mounin, J. Catford ou A. Ljudskanov qui, dans le titre même de leurs ouvrages, affichent des prétentions théoriques évidentes. En fait, tous trois rejettent la traduction dans le domaine de la linguistique pour les deux premiers, dans celui de la théorie de l'information dans le cas du dernier. Il n'est donc pas question de donner au phénomène un statut épistémologique autonome. À l'opposé de l'ensemble des analyses présystématiques, on trouve les 'artisans' de la traduction ${ }^{2}$ tirant de leur pratique un certain nombre de réflexions plus ou moins argumentées. Notre préférence va indéniablement à ces derniers qui ont l'immense mérite d'envisager la traduction globalement comme une opération sui generis (ainsi E. Cary, poussant cette position à l'extrême, ne traite pas de la traduction mais des traductions tirant leur spécificité du type de textes sur lesquels elles s'exercent) au lieu de la généraliser ou de la catégoriser inutilement comme les théoriciens. On trouve finalement, dans les ouvrages plus ambitieux de E. Nida ou J.-P. Vinay \& J. Darbelnet, des tentatives de compromis entre ces deux attitudes : à partir de sa pratique de traducteur de la Bible, Nida tente de dériver une théorie de l'équivalence sémantique tandis qu'à l'opposé, Vinay et Darbelnet s'appliquent à transposer les théories linguistiques de Bailly dans une stylistique comparée du français et de l'anglais. Loin de synthétiser les apports des 'artisans' et des 'théoriciens', on assiste, dans

1. Edmond Cary (1963): «L'indispensable débat», Quality in Translation, ed. E. Cary \& R.W. Jumpelt, Oxford, Pergamon Press, p. 47.

2. Cf. par exemple la majorité des articles dans le recneil de R. Brower (1966) : On Translation, New York, OUP. 
les deux cas, à des réductions inacceptables : une formalisation théorique insuffisante chez Nida, une pratique traductive totalement incontrôlée dans les exemples de traduction de Vinay et Darbelnet ${ }^{3}$.

Une coupure très nette sépare ces études des deux ouvrages qui ont non seulement déclenché mais aussi largement nourri cette recherche. Nous les diviserons également dans un souci de schématisme en 'visionnaires' et 'praticiens'. C'est aux premiers que nous devons de nouvelles orientations en traductologie. H. Meschonnic et, à un degré moindre L. Robel, ont su tirer la traduction $\mathrm{du}$ marasme épistémologique dans lequel elle se perdait, et redéfinir le rôle d'une pratique dans l'écriture et la culture. À J.R. Ladmiral, nous devons des stimulations moins violentes mais des analyses rigoureuses et fouillées sur les problèmes théoriques de la traduction, sa place dans l'institution pédagogique ainsi que l'esquisse d'une définition conceptuelle centrée sur la notion de connotation. Les uns et les autres se retrouvent curieusement à travers les notions voisines de 'praxéologie' (Ladmiral) et de 'pratique théorique' (Meschonnic), dans leur commune recherche d'une pratique coupée ou confondue avec sa théorisation. Notre recherche consistera à explorer cette suture épistémologique et à tenter de retendre le lien entre théorie et pratique. Si l'on ne saurait encore préjuger d'une éventuelle théorie des homologies, il est urgent de démasquer et de redéfinir les conditions exactes dans lesquelles s'exerce l'opération traduisante.

\section{LES 'IMAGES' IDÉOLOGIQUES DE LA TRADUCTION}

Les errements constatés dans les travaux récents sur la traduction sont la conséquence directe d'un profond travail d'occultation idéologique visant à déplacer le rapport entre la réalité objective du phénomène et le champ conceptuel permettant d'y accéder. Ce masquage est nettement perceptible à travers l'usage qui est fait des notions de 'traduction', 'traducteur', 'traduire'. La traduction est conçue, tantôt tour à tour, tantôt simultanément, comme 'processus', 'agentivité' ou 'produit'. Il semble même que, grossièrement, ces trois conceptions se soient échelonnées à différentes périodes de l'histoire, ce qui ne veut pas dire qu'elles sont 'dépassées' de nos jours. Ces trois attitudes ne se succèdent pas, elles coexistent sous des formes diverses à notre époque.

Cette fragmentation du processus au niveau de la langue s'accompagne également d'une déformation de l'élément ainsi isolé, mettant en évidence les diverses modalités d'un assujettissement de la fonction à des fins socioéconomiques.

La traduction comme produit est l'émanation directe d'une conception utilitariste du langage. Elle a connu, outre la banalisation excessive de la traduction technique, des complications technicistes dans les projets successifs de traduction mécanique ou, plus subtils, dans la traduction dite 'archéologique'. Il reste que, dans tous les cas, la traduction est un objet, hyper-objet pour le traducteur-savant ou objet monnayable du monde des affaires, mais objet tout de même coupé de toute pratique et de toute insertion culturelle ou historique.

3. Cf. dans Vinay \& Darbelnet, Stylistique Comparée, les traductions-modèles de l'Appendice III. 
La traduction comme pratique d'un traducteur (artisan/artiste selon les cas) situe le produit entre une impossible perfection et la nécessaire dévaluation que déclenche l'opération traduisante. Le traducteur est un agent indispensable mais impur, il introduit un 'bruit' certes réductible mais incompressible dans le transfert de l'information. Ce 'bruit' est inversement proportionnel à sa 'transparence', c'est-à-dire, en clair, à sa marginalisation ou à son effacement pur et simple dans la traduction dite 'universitaire'.

La réduction de la traduction au processus (introduite plus récemment par les adeptes de la machine à traduire et les 'sémanticiens' de la traduction), banalise le produit tout en supprimant ou minimisant l'intervention du traducteur. En fait, la boucle semble bouclée et, sous couvert d'une systématisation, c'est l'opération traduisante dans son intégrité qui se trouve une nouvelle fois escamotée.

Ces déformations successives aboutissent, on le voit, à une dévaluation du phénomène, à l'occultation de l'une ou l'autre de ses composantes, à leur systématique atomisation. On s'aperçoit, à l'issue de cette exploration préliminaire, qu'on court toujours le risque d'aborder un phénomène linguistique à travers les leurres conceptuels du langage, qu'on se débat avec des mots qu'il convient de reconfirmer ou de disqualifier.

Il n'est pas inutile, à cette fin, de passer en revue les 'images' historiques de la traduction, phénomène archéologique qui semble coexister avec les pratiques les plus archaïques de l'humanité. Ainsi, à l'origine, la traduction n'a pas d'image. Dans les sociétés primitives et les formes antiques des sociétés occidentales, la traduction est frappée d'interdit; la traduction, pratique dangereuse et suspecte, n'existe pas et le traducteur se voit exclu de la communauté car il représente l'être ambigu (répondant à une double allégeance) et, à ce titre, tombe sous le coup de l'interdit. Les premières traductions sont liées à la propagation des religions faisant éclater les limites tribales. Alors que le code social s'institue dans la diversité, la parole divine, inscrite dans la Lettre du Texte sacré, ne saurait se déformer dans son transfert vers les langues 'vulgaires'. La seule traduction envisageable est un démarcage littéral, une copie du texte initial. Plus tard, l'essor des sociétés marchandes du XVI ${ }^{e}$ au XVIII' siècles, encourage un nouveau type de traduction: la 'traduction-annexion' qui consiste à réécrire et à normaliser le texte étranger par la traduction. Les tracluctions de Voltaire ou de Pope en sont un exemple frappant. La civilisation triomphante des marchands a besoin de miroirs pour refléter sa puissance. La période romantique verra le retour à une conception littéraliste de la traduction où il ne s'agit plus alors de disséminer le texte sacré mais d'accéder à l'irréductible différence des autres cultures; les romantiques européens lissent les auteurs étrangers dans le texte et n'admettent que la plus littérale des paraphrases. L'avènement de l'ère industrielle voit s'imposer un clivage entre traduction poétique et traduction technique; l'une, réputée irréalisable, sera rejetée à la périphérie de l'univers artistique, l'autre, ainsi vidée de toutes composantes expressives et culturelles, se prêtera sans difficulté à des opérations de transcodage. 
On voit, au terme de ce rapide panorama historique que chaque 'image' représente la projection dans le champ idéologique d'un état déterminé des rapports entre cultures. Chaque nouvelle configuration historique secrète une nouvelle image conceptuelle qui a la double fonction de représenter et de masquer l'insertion de la traduction dans le jeu des forces économiques en présence.

Comme souvent, à l'intérieur des formations idéologiques, les différentes conceptions engendrées dans l'histoire ne se succèdent pas mais coexistent à chaque époque de façon cumulative. Elles se structurent et se positionnent les unes par rapport aux autres sans pour autant sortir du discours d'une période donnée. En passant en revue ces différents 'conglomérats' idéologiques, notre souci sera non seulement de nuancer les 'images' définies précédemment, mais également de souligner les contradictions qu'elles renferment. Car, l'image, définie comme déformation, entraîne nécessairement la contradiction. dage:

La première conception représente la traduction comme simple transco-

«When I look at an article in Russian I say: 'This is really written in English, but it has been coded in some strange symbols. I will now proceed to decode.' $»^{4}$

Cette dissociation caricaturale de la forme (code véhicule, truchement, etc.) et $\mathrm{du}$ sens (contenu) autorise conjointement une traduction mécanique et, plus généralement, une traduction en deux temps : traduction selon le sens précédant une traduction selon le style, comme dans les travaux de C. Taber et E. Nida. Il est curieux de constater que cette normalisation de l'opération traduisante est à la source de la notion d'intraduisible, déplacée, loin de la transparence du 'sens', dans les périphéries stylistiques de la poésie.

Une autre représentation parasite célèbre la transparence du traducteur et ses variantes moralisatrices, la fidélité, l'humilité ou le respect du texte initial. Mais il est facile de voir que c'est cette même conception qui a engendré simultanément l'image traditionnelle du 'traducteur-traitre' et celle, diamétralement opposée, du 'traducteur-esthète', à la fois monstre de savoir et abîme d'humilité. L'essentiel, comme précédemment est de déplacer le problème du sens et le rôle du traducteur dans les phénomènes culturels.

Une troisième image représente la traduction comme une 'écriture seconde' (parasite, décalée, placée au deuxième degré, etc.); une écriture qui se doit d'être neutre, exacte et impersonnelle ce qui, par voie de conséquence, laisse passer dans les meilleures traductions : réductions, élucidations, désambiguïsations, destinées à normaliser le texte-source. Le texte-cible doit être 'lisible' dans la langue cible; il faut à tout prix effacer une désastreuse impression de 'traduit'.

Finalement, la traduction doit être libre et indépendante de son public, écrite dans un vide culturel, et idéalement destinée à un public de lecteurs privilégiés alors qu'il ne fait aucun doute qu'elle est toujours orientée vers un destinataire quand elle n'est pas, dans le cas de la traduction du commerce, tout bonnement réécrite dans les maisons d'édition. Les traducteurs étant, c'est bien connu, gens de cabinet et non de plume.

4. W. Weaver (1955): Machine Translation of Languages, ed. Locke \& Book, New York, p. 48. 
Les tensions et les contradictions dans les représentations de la traduction accomplissent une double fonction : $1^{\circ}$ normaliser l'Étrange $(r) ; 2^{\circ}$ marginaliser le phénomène traductif. En fait, il s'agit, sur un plan plus large, de dissimuler la complexité des rapports entre inconscient, langue et culture et de minimiser l'importance d'une fonction dans l'économie des formations idéologiques.

Il est temps de retrouver une vision correcte des phénomènes tels qu'il nous est donné de les apercevoir - i.e. non naïvement - et, en premier lieu, d'observer et, peut-être, découvrir (au sens étroit de ce terme) les caractéristiques de la situation de traduction.

\section{ÉLÉMENTS CONSTITUTIFS DE LA SITUATION DE TRADUCTION}

L'une des occultations évidentes du discours contemporain consiste à reléguer la traduction au domaine de l'écrit. La traduction est essentiellement une opération livresque. Il convient de la resituer dans l'ordre de l'oral comme une configuration essentielle de l'acte de communication, non plus transcription mais activité de signification. Il est urgent, également, d'envisager le processus dans sa totalité; c'est-à-dire englobant tout à la fois un agent et un produit, mais également une situation de traduction et un destinataire de la traduction, à la fois point de départ et aboutissement de l'opération.

À partir d'un schéma simplifié de la communication que l'on peut représenter comme suit :

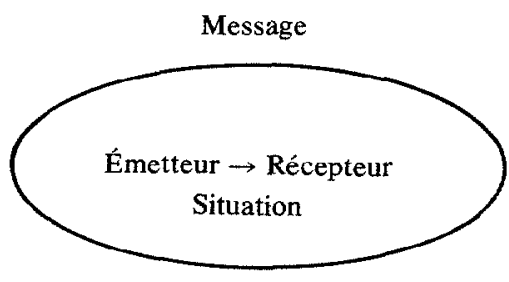


La situation de traduction se définit comme suit pour l'énoncé suivant:

A : [alor כ̃ $s \in m$ ?[

B : Comment?

A : Je veux dire, on les plante, ces salades? ${ }^{5}$

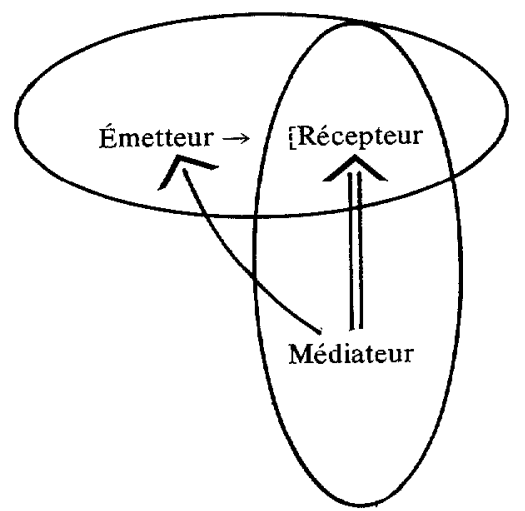

où l'on peut isoler les particularités suivantes:

- la traduction s'inscrit fondamentalement dans la rupturelinterruption (imaginaire ou simulée, totale ou partielle, présente ou diachronique) de l'échange normal de communication due à la non-correspondance des codes Émetteur et Récepteur. Pour que la traduction ait effectivement lieu, on présuppose un certain nombre de conditions :

- un désir de communiquer de la part de l'Émetteur sinon l'échange se termine dans l'hermétisme qui est à l'opposé de la traduction.

- la portée de la situation de traduction est radicalement modifiée. Elle consiste à prendre en charge la totalité d'une situation déjà réalisée, ce qui infléchit sensiblement les paramètres de cette situation adventice :

- l'énoncé est orienté prioritairement vers le récepteur et son besoin d'information,

- la situation de traduction est théoriquement neutralisée,

- la fonction expressive du médiateur est également théoriquement minimisée, - le rôle des participants est considérablement modifié :

- la communication s'adjoint un tiers, médiateur compétent dans les codes mis en présence.

- la participation du médiateur est neutralisée et l'importance du Récepteur se trouve hypertrophiée.

- la situation de traduction présuppose non seulement, comme on l'a déjà souligné, la pluralité des codes mais également une hiérarchisation entre

5. Nous ayons choisi à dessein une situation de paraphrase intralinguale dans le cas d'une ambiguité homophonique et lorsque le destinataire est son propre traducteur. 
code Fort (code traduit) et code Faible (code dans lequel on traduit); cette hiérarchisation (sans valorisation, du moins dans l'état brut dans lequel nous envisageons les faits), plus ou moins figée sur le plan social (situations de diglossie), demeure extrêmement variable sur le plan individuel et résulte toujours des variations de compétence du Récepteur.

- cette hiérarchisation s'accompagne, corrélativement, d'une certaine co-pénétration des codes allant du simple infléchissement jusqu'à l'emprunt pur et simple. Il est nécessaire de postuler que le locuteur du code Fort adapte nécessairement son langage et que le Récepteur accepte de se laisser influencer par un message à priori étranger à son système habituel de références. Cette osmose prendra, sur le plan des langues, les formes diverses d'ingérences (calques, emprunts, sabirs) ou d'assimilations plus ou moins accomplies dont la traduction semble être le facteur essentiel.

Une série de conclusions concernant le phénomène de traduction sont ainsi mises en évidence :

- la traduction comme re-énonciation,

- l'orientation prioritaire vers le destinataire,

- la hiérarchisation des codes,

- la co-pénétration des systèmes,

autant de facteurs dont il faudra tenir compte dans l'élaboration d'une théorie de la traduction ainsi que dans la définition d'une pratique.

\section{ÉLARGISSEMENT DE LA NOTION DE TRADUCTION}

Il serait inutile de décrire l'opération traduisante si l'on ne saisissait pas en même temps sa fonction essentielle dans la communication humaine.

Déjà R. Jakobson, transposant les théories de C. Pierce, assignait à la traduction une fonction d'interprétance assurant le glissement et la conversion des différents codes les uns dans les autres. Il y a pour lui trois types de traductions : intralinguale, ou reformulation, interlinguale ou traduction proprement dite et intersémiotique ou transmutation dont $\mathrm{E}$. Benveniste ${ }^{6}$ a montré qu'elle se réduisait à une relation de dérivation, illustrant de ce fait l'une des propriétés fondamentales du langage: être l'interprétant de tous les autres systèmes linguistiques et non-linguistiques. Restent les deux autres types de traductions dont Jakobson se sert pour illustrer la fonction cognitive du langage et les problèmes de traduisibilité dans les langues naturelles. En simplifiant, la traduction serait l'instrument privilégié des sciences du langage destiné à mettre en évidence une relation fondamentale: «Equivalence in difference [...] the cardinal problem of language and the pivotal concern of linguistic ${ }^{7} \gg$. Un problème, une préoccupation, nous soulignons à dessein ce terme pour montrer une nouvelle fois que la traduction se trouve écartée de son rôle de fonction et ramenée à celui d'opération.

On ne saurait non plus se contenter d'une fonction de normalisation des ratés de l'échange communicatif telle que notre étude strictement descriptive

6. Problèmes de linguistique générale, II, chap. 3, "Sémiologie de la langue».

7. R. Jakobson (1966) : "On Linguistic Aspects of Translation», On Translation, ed. R. Brower, New York, OUP, p. 233. 
semblerait le faire accroire. On traduirait - c'est-à-dire, on changerait de code, à supposer que cela soit possible, utile - dès l'instant où l'on cesserait de comprendre ou d'être compris. Une telle conception est difficilement tenable; elle suppose, entre autres conditions, que l'instant où la communication s'interrompt apparaîtrait à l'évidence aux partenaires concernés, que la communication une fois rétablie ne serait plus perturbée ni surtout affectée de l'avoir été et, plus simplement, que la traduction aurait fait son office sans bavure ni résidu.

Bien qu'une grande partie de ces problèmes soient loin d'être résolus, en l'état présent des connaissances, il apparaît préférable de situer la traduction dans le cadre d'une théorie de l'échange verbal où la compréhension et la noncompréhension se trouvent dans un rapport constant de complémentarité. Nous ferions volontiers nôtre une remarque parenthétique de A. Culioli s'adressant à une assemblée de littéraires: «Il y a toujours perte dans l'échange verbal et l'on pourrait dire que, en fin de compte, la compréhension est, dans la communication, un cas particulier du malentendu ${ }^{8}$.

L'élargissement du concept de traduction amorcé par R. Jakobson, permet de dénoncer le mythe tenace de la transparence de la langue natale par opposition à l'opacité maximale de la langue étrangère. Une fois de plus, le masquage idéologique n'a pas totalement occulté une donnée réelle mais déplacé, à des fins qui lui sont propres, l'opacité constitutive du langage dans l'Étrange ou l'Ineffable du discours poétique qui, on le verra plus tard, fait problème dans la définition des conditions de traduisibilité.

Si l'opacité ou plus exactement l'équivoque (car il faut être deux - au moins - pour équivoquer et l'on n'équivoque pas à partir de n'importe quoi...) sont inscrites dans le langage; si, par ailleurs, les différentes situations de blocage inter- ou intra-linguales ne sont pas de nature mais seulement de degrés, alors la traduction remplit une fonction capitale dans la communication que nous nommerons, en adaptant la terminologie de $\mathrm{E}$. Benveniste, la fonction d'Interprétance qui recouvrirait, du point de vue qui est le nôtre, les deux autres relations 'sémiologiques' de dérivation et d'homologie.

La fonction d'Interprétance est une propriété inhérente au langage. La traduction, en tant que pratique linguistique, est une manifestation de cette fonction, non distincte en nature des autres pratiques dont elle est traditionnellement disjointe. Comme il n'y a pas de langue in abstracto (idéalement formalisable), mais des systèmes imbriqués, historiquement, psychologiquement et socialement déterminés, parler une langue, s'exprimer dans sa langue revient à évoluer entre tous ces systèmes et sous-systèmes. La fonction d'interprétance correspond directement à l'essentielle complexité de la langue, construction systématique de systèmes multiples. Il est de la compétence de tout locuteur d'une langue de pouvoir conjointement signifier et convertir, entièrement ou partiellement, à partir des codes de signification auxquels il peut avoir accès.

Les deux fonctions de Signifiance et d'Interprétance apparaissent étroitement corrélatives, comme l'a si justement formulé G. Steiner : "Any model of

8. A. Culioli (1969) : «Un Linguiste devant la critique littéraire», Actes de la S.A.E.S., Paris, Didier, p. 64 . 
communication is at the same time a model of trans-lation, of a vertical or horizontal transfer of significance ${ }^{9} \%$. On pourrait grossièrement représenter leurs rapports comme des grandeurs inversement proportionnelles ne pouvant être nulles ni l'une ni l'autre. Il y aurait toujours asymptotiquement une part de signifiance dans la traduction placée à l'une des extrêmes de la courbe : ainsi, l'activité du traducteur n'engendre pas un résidu, un 'bruit' sémantique parasite mais une authentique part de signification; de même, à l'autre bout, il n'y aurait pas de signifiance sans une part, aussi minime soit-elle, d'interprétance; le locuteur (et son interlocuteur par une espèce de choc en retour) traduit et se traduit (sans qu'il y ait nécessairement correspondance entre les deux produits de part et d'autre) en même temps qu'il signifie.

On peut, en faisant jouer ces deux variables, caractériser différents types d'interprétations présents à tout instant dans le discours :

- au niveau le plus bas d'interprétance, on peut placer les deux pôles de la communication intralinguale que $\mathrm{C}$. Fuchs et $\mathrm{P}$. Le Goffic décrivent, dans lleur évocation de la linguistique soviétique comme "le 'système de traduction' du locuteur... et celui de l'auditeur... ${ }^{10}$, mettant ainsi fortement en évidence, lle disparate des conditions d'appropriation du système à l'intérieur d'une même langue;

- plus haut dans l'échelle d'interprétance, on situera la paraphrase stricto sensu ainsi que les diverses reformulations, définitions, gloses, circonlocutions, etc. et, plus généralement, toute activité de réglage à l'intérieur d'un même code (cf. notre exemple dans le développement précédent);

- un degré plus élevé dans l'interprétance caractérise l'adaptation réglant la conversion entre les différents sous-systèmes objectivement repérables (idiolectes, dialectes, technolectes, etc.) qui forment la langue d'une communauté donnée;

- l'étape suivante introduit la conversion ou mutation des codes sémiotiques les uns dans les autres et leur interprétation dans la langue;

- on arrive enfin, à l'autre bout de la courbe, à la traduction proprement dite entre langues naturelles.

Cette fonction une fois brièvement décrite à travers ses divers domaines d'application ainsi que les conditions dans lesquelles elle s'applique, il nous reste à définir ses limites : d'une part, sur quoi et dans quelle mesure elle peut s'exercer et, d'autre part, dans quelles conditions et jusqu'où se réalise le processus de traduction. On verra que ces deux problèmes se trouvent réunis dans l'examen des conditions de traduisibilité.

\section{TRADUISIBLE ET INTRADUISIBLE}

Ces deux notions placent l'opération traduisante curieusement dans l'axe du potentiel et, en même temps, dans celui du prospectif. Le traduisible (ou l'intraduisible) l'est-il essentiellement, dans certaines conditions et certains types

9. G. Steiner (1975): After Babel, Londres, OUP, p. 45.

10. C. Fuchs \& P. Le Goffic (1975) : Initiation aux problèmes des linguistiques contemporaines, Paris, Hachette, p. 104. 
de discours ou bien, est-il directement lié à une pratique? Autrement dit, y a-t-il un intraduisible de la langue et/ou un intraduisible du traducteur? La distinction entre traduisible et intraduisible est loin d'être claire: traduisible et intraduisible sont-ils vraiment complémentaires? L'intraduisible est-il le contraire du traduisible? Curieusement, le traduisible ne semble jamais faire problème : c'est 'ce qui est traduit quand on traduit'. Dans l'évidente tautologie de la formule passe une impérieuse nécessité pratique cherchant à faire oublier le caractère problématique d'une fonction.

Le vrai débat n'est en général jamais abordé. On se place d'emblée dans l'aporie de l'objection préjudicielle: on énonce, comme G. Mounin, les raisons historiques et théoriques 'prouvant' l'impossibilité de la traduction tout en concédant non seulement la réalité tangible du phénomène mais aussi sa nécessité pratique. S'il est utile de parcourir l'argumentation de J.-R. Ladmiral ${ }^{11}$ reprenant terme à terme tous les aspects du débat, il est indispensable de dénoncer, une fois de plus, un nouvel 'oubli' nettement référencié au niveau idéologique. L'intraduisible n'est que l'image inversée de la traduction idéale sans résidu dont on a vu qu'elle servait de fondement aux projets de traduction automatique, l'une et l'autre de ces notions se servant mutuellement de repoussoir dans une opposition non dialectique. En fait, il n'y a pas de réelle antinomie entre les deux termes mais une contradiction déguisée dont on peut représenter les avatars sous la forme d'un carré sémiotique où les flèches indiquent des oppositions terme à terme :

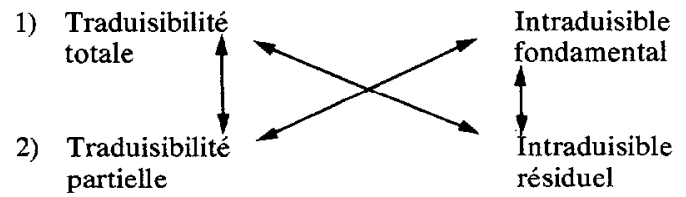

Dans la première ligne, on reconnaît la version maximaliste du masquage cherchant à gommer l'effet d'étrangeté, dans l'autre (ligne du bas), la version minimaliste visant à réduire et refouler l'Étrangeté. Dans les deux cas, on retrouve la même attitude négative envers l'Étrange/l'Autre qui doit être nié ou normalisé. C. Roy résume parfaitement cette résistance en resituant le phénomène sous l'angle psychologique :

En vérité, la traduction n'apparaît impossible que comme apparaît inaccessible la connaissance de soi. Car nous sommes capables de traduire ce dont nous avons besoin. Car nous refusons de traduire ce à quoi notre surdité intérieure oppose une résistance, ce qu'elle censure ${ }^{12}$.

Plus récemment, le débat sur les conditions de traduisibilité s'est développé sur un champ plus limité, à partir d'observations scientifiques. Développant une conception strictement fonctionnelle de l'opération traduisante, il était prévisible que $R$. Jakobson après une pénétrante étude des phénomènes d'équivalence dans la traduction interlinguale, soit amené à isoler une zone d'intraduisible absolu qu'il situe dans le discours poétique: «...Poetry by

11. J.-R. Ladmiral, Traduire, chap. 3.

12. C. Roy, «Le Vain travail de 'traduire' la poésie chinoise», Change, 19, p. 157. 
definition is untranslatable ${ }^{13}$. Il y aurait donc un intraduisible partiel que Jakobson situe dans la paronomase mais que d'autres placeront dans le 'style' ou la 'connotation'. À travers toutes ces variantes il est clair que le phénomène poétique n'est pas situé dans la langue mais en marge de la langue, dans l'écart par rapport à une norme introuvable. En réalité, il s'agit de minimiser le processus d'écriture et de marginaliser l'activité de l'écrivain. La poésie ne se situe ni au-delà ni en marge mais en plein coeur de la langue dont elle explore les possibilités au même titre que toutes les autres pratiques discursives.

Si l'on se tourne du côté de la norme de référence, logiquement idéalement traduisible, il est permis de douter de l'existence d'une hypothétique langue courante, véhiculaire ou ordinaire. Lorsqu'elle est un peu mieux définie ou lorsqu'on essaye de faire sauter ces 'termes-écrans', on découvre que la norme recouvre tantôt le domaine de la langue parlée, tantôt le discours scientifique. Il n'est peut-être pas inutile de rappeler que la langue parlée n'est pas une catégorie homogène mais un lieu de rencontre de différents parlers à travers lesquels évoluent les locuteurs. De même, il faut dénoncer l'opinion largement répandue que le langage de la technique ou celui de la science seraient strictement véhiculaires et passeraient sans résidu d'une langue à l'autre. En fait, comme l'a montré J.-R. Ladmiral ${ }^{14}$ à partir de son expérience de la traduction philosophique, la langue spécialisée pose de redoutables problèmes d'homologies lexicales de nature à redistribuer le champ conceptuel de la langue-cible.

Tout est donc potentiellement traduisible et rien ne saurait être traduit si l'on accepte les analyses de Sapir et Whorf qui ont montré que les langues issues de systèmes socio-économiques différents ne sauraient, par conséquent, être mutuellement convertibles. Nous voilà donc replongés dans l'aporie de l'objection préjudicielle: les langues seraient intraduisibles par leur nature même et un éventuel recours à d'hypothétiques universaux du langage ne saurait tirer le traducteur de cette inconfortable - et absurde - situation.

La contradiction n'est pourtant pas insurmontable. L'hypothèse Sapir/ Whorf est infiniment préférable à tout autre tendant à établir un rapprochement ou une parenté entre les langues. Les langues sont fondamentalement différentes et c'est pour cela qu'on les traduit. Si le traducteur a tout intérêt à sortir de son aire linguistique pour pratiquer la traduction dans ou à partir de langues éloignées de la sienne, c'est uniquement pour instituer ce rapport de totale étrangeté entre les langues considérées comme voisines.

L'intraduisible se réduit finalement à du non-traduit, c'est-à-dire comme le produit de sujets-traducteurs historiquement situés au sein d'une culture donnée. Il n'y a donc pas fondamentalement d'intraduisible mais des limites liées à «l'aventure historique d'un sujet» et, poursuit $H$. Meschonnic :

Pour une œuvre donnée dans un rapport interlinguistique-interculturel donné, l'interraction des poétiques et la ré-énonciation historique peuvent ne s'être pas encore produites, peuvent ne pas se produire. L'intraduisible comme texte est alors l'effet culturel résultant de ces raisons historiques. L'intraduisible est social et historique, non métaphysique (l'incommunicable, le mystère, le génie) ${ }^{15}$.

13. R. Jakobson, «On linguistic Aspects of Translation», On Translation, op. cit., p. 238.

14. J.-R. Ladmiral, Traduire, op. cit., 6.2, p. 216 et ss.

15. H. Meschonnic (1973) : Pour la poétique, II, Paris, Gallimard, p. 309. 
Nous renvoyons le lecteur sans commentaire à l'ensemble des analyses de H. Meschonnic dans Pour la Poétique II et notamment, "Propositions pour une poétique de la traduction" et "D'une linguistique de la traduction à la poétique de la traduction».

Il s'ensuit que le traduit, de par sa nature de texte historiquement situé, est nécessairement révisible et donc infiniment ré-énonçable. La traduction ne connaît ni limites ni butoirs indépassables. Chaque moment historique à travers ses sensibilités particulières refera ses propres traductions.

Loin d'être un simple moyen de contact et d'échange, la traduction «dedans/dehors d'une langue et de sa littérature » (Meschonnic 1973: 360) règle les phénomènes osmotiques qui accompagnent les rapports entre pouvoirs économiques et politiques. S'opposant à la fois au purisme (du type Parlez-vous Franglais? d'Etiemble) et au laxisme linguistique de la langue contemporaine, il s'agit d'instituer, par la traduction, une autre relation entre les cultures : les langues sont condamnées à s'enrichir de toutes les traductions qu'elles sont capables de produire ou à être progressivement annexées.

Une conception différente de la traduction est donc nécessaire pour concilier, à travers la fonction d'Interprétance, le rôle fondamental d'un Sujet inscrit par sa pratique dans la contradiction des langues-cultures, clairement soulignée par J.C. Vegliante: "Traduire, c'est tenter de dire autrement ce qui dans chaque langue ne peut être dit autrement» ${ }^{16}$. Des notions comme la 'compensation', 1'interprétation minimale' ou le 'contre-sens minimal' proposées par J.R. Ladmiral, paraissent encore bien conservatrices et toutes pénétrées de l'échec auquel s'expose nécessairement le traducteur. Elles semblent également impliquer une espèce de bricolage ponctuel condamnant le traducteur à 'rafistoler' ses traductions pour masquer le scandale de la non-conformité des langues. Une autre possibilité, l'Équivalence, avère la traduction par 1'interchangeabilité des situations qui renvoie le traducteur à l'impossible compromis du Même et de l'Autre et replace la traduction dans la glorieuse incertitude de l'intraduisible.

Une nouvelle fois, $H$. Meschonnic propose le concept le plus utile avec le Décentrement :

Mais, transportant dans la pratique linguistique le décentrement dont parlait Massignon, on peut le situer à deux niveaux : celui de la langue, celui d'un texte dans sa langue. Si le découpage du réel n'est pas le même d'une langue à l'autre, la traduction, suivant l'évolution même de l'anthropologie, n'a plus à être annexion mais rapport entre deux cultures-langues : non la disparition fictive de l'altérité, mais la relation dans laquelle on est, ici et aujourd'hui, situé, par rapport au traduit. La traduction n'est plus alors la 'Belle Infidèle' mais la production et le produit d'un contact culturel au niveau des structures mêmes de la langue ${ }^{17}$.

La définition, il faut le convenir, reste encore vague et difficile d'application. Nous lui préférerons la notion d'Homologie(s) décrivant une mise en relation de système textuel à système textuel entre langue-source et langue-cible plutôt

16. J.C. Vegliante, "Traduction, traduction des dialectes, interlangues», Langues Modernes, LXXIV, I, p. 80.

17. H. Meschonnic, Pour la poétique, op. cit., p. 413. 
qu'une nouvelle répartition des valeurs par rapport à un centre dont nous ne voyons guère l'application en linguistique. La recherche des homologies amène le traducteur à analyser et à structurer à tous les niveaux les valeurs du message en langue-source et à les 'simuler' dans le système de valeurs de la languecible. Il ne s'agit donc ni de convertir la langue-cible, ni d'annexer la languesource mais d'explorer la contradiction qui rapproche et oppose le texte traduit et sa traduction. Le traducteur ne cherchera pas tant à reconstruire, transférer, simuler qu'à élaborer son texte sous la double contrainte d'une lecture et d'une écriture. Il s'engage non seulement à se lire et à s'écrire tant au départ qu'à l'arrivée mais il se place, de surcroît, dans un rapport de tension visant à articuler deux langues-cultures. Le traducteur ne saurait être conservateur, comme le voudrait J.R. Ladmiral, il est condamné à être novateur, il n'a pas d'autre possibilité.

\section{LES PARAMÈTRES DE L'OPÉRATION TRADUISANTE}

La traduction est une pratique. On ne le répétera jamais assez. Mais, on voit mal pourquoi elle devrait se cantonner dans une 'praxéologie' coupée de toute théorie et analysable dans ses seuls produits comme le voudrait J.R. Ladmiral ${ }^{18}$ ou devenir, mystérieusement, pour H. Meschonnic, une 'pratique théorique' 19 . De même que la psychanalyse, la médecine ou la psychologie, l'ensemble des pratiques en sciences humaines ne saurait se concevoir sans une certaine conceptualisation théorique. La traductologie ne fait pas exception.

Si l'on passe en revue les constructions théoriques qui ont été proposées, on peut mettre rapidement de côté le concept de transcodage qui fonde la quasitotalité des études soucieuses de donner un arrière-plan méthodologique à leurs remarques. Il est facile de reconnaître une conception instrumentale du langage fondée sur l'universalité du sens sous la diversité des véhicules. Une variante moderne reparaît dans les travaux de A. Ljudskanov, basés sur la théorie de la communication et la notion d'Interlangue (langue de transfert entre la languesource et langue-cible) qui semble accumuler, dans le vague méthodologique, tous les défauts des précédents systèmes. Les modèles les plus élaborés (Vinay/Darbelnet ou Catford) s'appuient sur la notion d'équivalence mettant en rapport paraphrastique des énoncés reconnus comme équivalents en situation selon le schéma de Vinay/Darbelnet :

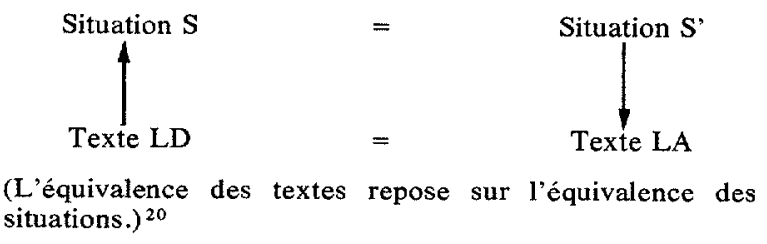

L'équivalence du texte et de sa traduction serait validée par le recours à la référence. Mais on comprend mal pourquoi ce qui est conçu comme différent

18. J.-R. Ladmiral, Traduire, op. cit., pp. 189-90.

19. H. Meschonnic, Pour la Poétique, op. cit., p. 305.

20. J.P. Vinay \& J. Darbelnet (1958): Stylistique Comparée du français et de l'anglais, Paris, Didier, p. 22 
au niveau des catégories de la langue se retrouverait comparable dans l'extralinguistique. Sans même parler du problématique rapport de tout énoncé à sa situation ni des conditions d'équivalence entre deux énoncés, il est aisé de voir resurgir, à travers ces analyses, les leurres d'une conception spéculaire du langage, d'ailleurs complémentaire de l'illusion instrumentale dans la perspective utilitariste.

Le meilleur garant d'une éventuelle traductologie semble reposer sur une théorie généralisée des homologies. Le principe des familles de paraphrases ou faisceaux d'énoncés reliés par un ensemble de valeurs nettement discriminées devrait permettre de situer l'énoncé traduit dans son double rapport différentiel avec le texte et le système de la langue-source. Il est, en plus, le seul qui permette à la culture et aux systèmes connotatifs du traducteur de s'inscrire pleinement dans sa pratique. La traduction comporterait deux temps : une mise en relation du texte à traduire avec ses homologues en langue-cible (ce qui, par voie de conséquence, amènera le traducteur, par un effet de recul à réactiver les associations paraphrastiques du texte-source) suivie d'une prise de décision motivée du traducteur. Sommes-nous ramenés, pour le coup, à l'image d'un traducteur artisan/artiste dont nous avions cru nous défaire? En réalité, il ne s'agit pas de minimiser ou d'éliminer le choix du traducteur mais d'éclairer sa prise de décision par une information théorique dont nous nous proposons d'explorer les domaines.

Pour des considérations pratiques, nous avons été amené à distinguer des niveaux d'analyse qui sont, dans la pratique du traducteur, étroitement imbriqués. Il n'est même pas sûr, si l'on en croit les travaux de P. Henry et A. Gresillon sur les relatives ${ }^{21}$, qu'il soit même utile de les envisager séparément. Cette distinction entre domaines linguistique, situationnel et socio-culturel nous a simplement permis de classer les apports venant de différents horizons en sciences humaines. En l'état de la recherche en traductologie, cette démarche nous parait inévitable; le chercheur est contraint de piller les sciences voisines et de 'bricoler' les concepts susceptibles de l'aider à définir ses propres stratégies exploratoires. Sa seule excuse sera de ne rien exclure a priori de façon à affiner les options offertes au choix du traducteur.

L'objectif prioritaire est de sérier les difficultés de façon à éviter définitivement le recours aux 'recettes', 'techniques' ou 'méthodes' de traduction auquel se trouve acculée toute pratique empirique. Il va sans dire qu'une attitude prescriptive ou techniciste irait à l'encontre de nos présupposés. Nous adopterons, à titre provisoire, une démarche heuristique assez proche de celle définie par J.-R. Ladmiral. ${ }^{22}$ sans ses a prioris épistémologiques. Il apparaîtra clairement que les réponses que nous apportons dans certains domaines ne dépassent pas le plan de l'esquisse et demandent un approfondissement considérable. Les orientations devraient pouvoir être conservées et servent dans notre pratique pédagogique à une réflexion méthodologique et pratique sur la traduction.

21. Langages, IX, mars 1975,37

22. J.-R. Ladmiral, Traduire, op. cit., p. 176, cf. aussi p. 194. 
C'est avec la linguistique que se sont établis les contacts les plus nombreux et les plus aberrants. Certains linguistes ignorent la traduction, d'autres la relèguent au rang des applications. Il existe, il est vrai, une différence radicale entre les objectifs et le champ épistémologique de la linguistique et de la traductologie. Perspective minimaliste dans le cas de la linguistique s'opposant à la traduction toujours placée dans la globalité des phénomènes. Les deux démarches ne sont pourtant pas divergentes et devraient nécessairement se rejoindre. Ainsi, la traductologie devrait profiter des travaux d'A. Culioli sur la dérivation de familles paraphrastiques d'énoncés. En élargissant le concept de prédication définie très largement comme 'mise en relation', il est possible d'envisager les unités lexicales comme des prédications stabilisées (prédication 'dépassée' dans le cas du substantif, prédication 'd'attente' dans le cas du verbe). Il s'agirait d'ensembles de relations, variables dans leur complexité selon les types de langues et plus ou moins stables dans leurs configurations, sur lesquels l'intervention de l'énonciateur est pratiquement nulle. L'analyse sémique donne une analyse aussi rigoureuse que possible de ces ensembles; ainsi, l'unité lexicale anglaise TIE pourrait se réécrire :

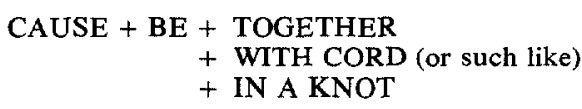

où les capitales représentent des fragments indissociables du sémantisme spécifique de TIE. Il est facile, dans un premier temps de la distinguer utilement de son homonyme :

$$
\text { BE + OF EQUAL SCORE }
$$

Sans entrer dans le difficile problème de la définition et du statut des traits distinctifs, on voit que se trouve également clarifié le problème de la paraphrase de définition et celui des parasynonymes et familles lexicales auxquels le traducteur se trouve constamment confronté. Ainsi, en filtrant diversement les traits isolés précédemment, il sera facile de dériver des termes tels que : CONNECT, BIND, LINK, KNOT, etc. mettant respectivement en valeur le processus, l'instrument et le résultat et situés dans un même rapport d'hyponymie avec TIE. On pourra ensuite faire jouer les proximités contextuelles pour discriminer des sens métonymiques comme :

- en contexte convergent, TIE prendra le sens de UNITE

$$
\text { - en contexte divergent, TIE s'apparentera à CONSTRAIN }
$$

On voit, du même coup, que disparaît l'opposition entre sens propre, littéral et sens figuré ou métaphorique; le figuré devient un effet rhétorique lié à l'intracontexte se diluant dans le problème plus large de la métaphorisation, elle-même régie par des contraintes d'ordre contextuel.

On pourra finalement dériver de la configuration de base les différentes potentialités grammaticales de l'unité lexicale TIE qui sont autant de lectures du sémantisme de base. Ainsi apparaîtront corrélés les différents usages de 
TIE (instrument, objet, nominalisé, état) et, de la même façon, TIED, TYING, TIED, TIER. On voit, également, qu'il doit être possible d'agréger à cette notion de 'prédication stabilisée' tout l'éventail des préfixes, suffixes, dérivations et compositions lexicales ainsi que les nominalisations formant autant de stabilisations syntaxiquement repérables de prédications dépassées en discours.

On peut, dans un deuxième temps, intégrer ces prédications stabilisées dans des prédications construites et référenciées en discours. Cette mise en relation par le biais de la prédication permet, sinon de supprimer, du moins de repenser le traditionnel partage entre lexique et syntaxe, variable selon la diversité des langues. Il est aussi possible d'envisager une mise en relation plus souple entre langue-source et langue-cible qui dépasse les limites étroites de l'unité lexicale et permette des homologies plus fines et plus nuancées.

Si les recherches en ce domaine sont encore peu avancées, il est d'ores et déjà possible de faire des distinctions pertinentes entre des énoncés considérés par Ladmiral comme exactement synonymes ou 'variantes libres' :

'La pluie ne cesse de tomber'

'Il ne cesse de pleuvoir'

où une analyse plus exacte distinguerait une opposition entre notion et processus que des contextes appropriés permettraient de discriminer, même si, dans certains cas limités, ces deux énoncés peuvent paraître interchangeables. Il va sans dire qu'une telle différence est capitale du point de vue de la traduction. De même, on peut amener l'apprenti-traducteur à analyser les différents éléments d'une famille de paraphrases que l'on peut envisager comme des homologues possibles de l'énoncé français: 'Des feuilles s'enroulaient autour des branches' :

Leaves were coiling round branches

Leaves coiled round branches

Leaves got coiled round branches

Leaves coiled themselves round branches

On s'aperçoit alors que si l'analyse linguistique ne dicte pas le choix du traducteur, elle réduit considérablement l'éventail des possibilités et motive fortement sa décision.

De la même façon se trouveront justifiées des études portant non seulement sur les articulations interphrastiques mais sur les micro- et macro-contexte. Il conviendra également d'envisager la dimension textuelle mais, dans ce domaine, on ne semble pas avoir dépassé le plan des observations et des taxinomies, mis à part les travaux de J.B. Grize et G. Vignaux.

Nous serons encore plus allusif, pour l'instant, dans l'examen des deux autres domaines. Le domaine situationnel devrait comporter trois relations fondamentales: la dimension interpersonnelle dans laquelle entrent la dialectique intersujet et les notions d'adressage et d'expressivité; la relation de l'énoncé à la situation faisant la part du dit et du non dit, de l'implicite et de l'allusif, etc. et la relation au message dans laquelle apparaîtront les effets poétiques (au sens jakobsonien), le choix des registres d'expression et la position sémio- 
tique de texte linguistique, sa place et ses rapports dans d'autres systèmes de représentation (cf. bande dessinée, publicité, etc.).

Le dernier domaine, socio-culturel, s'impose particulièrement au traducteur malgré les avertissements de Ladmiral : «On peut dire globalement que le fonctionnement sémantique de la langue est sans véritables frontières de classes ${ }^{22}$ !", directement battus en brêche par l'ensemble des travaux du groupe Langage et Société faisant apparaître, dans le discours l'importance des notions de rôle, statut, fonction; les groupes d'appartenance et les relations de classes; les codes, dialectes sociaux, et valeurs des différentes structures sociales.

Dans tous ces domaines, le traducteur aura intérêt à suivre au plus près les recherches les plus propres à éclairer sa pratique; de même, le traductologue devrait avoir son rôle à jouer dans l'articulation de ces différents domaines d'exploration trop souvent disjoints et parfois même en situation de conflit.

Préoccupation séculaire, la traduction, comme toutes les autres pratiques langagières, est marquée par la contradiction qui frappe tout discours : elle est toujours et en même temps une pratique et une réflexion sur cette pratique. Cette contradiction n'est-elle pas inscrite dans le langage même? Lorsqu'on 'traduit sa pensée', on cherche en même temps à signifier et à attirer l'attention sur la façon dont s'accomplit cet acte de signification. Les contradictions qui traversent le discours sur la traduction sont à la fois nombreuses et donc, dans une certaine mesure, inévitables. Elles conditionnent aussi bien l'ampleur de la réflexion contemporaine que l'absence de réelle continuité dans les constructions scientifiques proposées.

Notre étude s'est attachée à dépister et à démasquer les leurres culturels qui déforment le concept de traduction pour tenter de retrouver le phénomène traductif dans son originalité fondamentale. De cette exploration sont ressorties conjointement la notion d'Interprétance comme composante indissociable du processus de signification et la spécificité d'une Pratique Traductive. Ces deux domaines d'exploration et surtout leurs rencontres (les traductions que se donnent les cultures) ainsi que leurs correlations (la traductologie) devraient permettre de faire franchir à la recherche le 'seuil de scientificité' dont parle Michel Foucauld.

\section{BIBLIOGRAPHIE}

BENVENISTE, E. (1974) : Problèmes de linguistique générale, II, Paris, Gallimard. BROWER, R., Ed. (1966) : On Translation, New York, OUP.

CARY, E. and R.W. JUMPELT, eds (1963): Quality in Translation, Oxford, Pergamon Press. CATFORD, J.C. (1965) : A Linguistic Theory of Translation, Londres, OUP. CHANGE (Collectif) (1973) : «Transformer, traduire», 14, Paris, Laffont, Seghers. CHANGE (Collectif) (1974) : «La Traduction en jeu», 19, Paris, Laffont, Seghers. HOLMES, J.S., Ed. (1970) : The Nature of Translation, The Hague, Paris, Mouton. JAKOBSON, R. (1963) : Essais de linguistique générale, Paris, Minuit, Points.

LADMIRAL, J.-R. (1979) : Traduire : théorèmes pour la traduction, Paris, Payot.

23. Cf. les analyses pénétrantes de Ladmiral dans le chapitre intitulé «La Traduction et l'Institution Pédagogique» dans Traduire, op. cit., p. 23-82. 
LADMIRAL, J.-R., Ed. (1972) : Langages, 28, déc, Didier, Larousse.

LJUDSKANOV, A. (1969): Traduction humaine et traduction mécanique, fasc. 1 et 2 , Documents de linguistique quantitative, $\mathrm{n}^{\circ} 2$ et 4 , Association Jean-Favard.

MESCHONNIC, H. (1973): Pour la poétique II, Épistémologie de l'écriture, Poétique de la traduction, Paris, Gallimard.

NIDA, E. (1964) : Towards a Science of Translating, Leyde, Brill.

VINAY, J.P. et J. DARBELNET, 1958) : Stylistique comparée du français et de l'anglais, Paris, Didier. 